\title{
Influence of Inhomogeneity and Initial Stress on the Transient Magneto-Thermo-Visco-Elastic Stress Waves in an Anisotropic Solid
}

\author{
Mohamed Abdelsabour Fahmy \\ Faculty of Computers and Informatics, Suez Canal University, Ismailia, Egypt \\ E-mail: mafahmy2001@yahoo.com \\ Received July 14, 2011; revised August 16, 2011; accepted September 2, 2011
}

\begin{abstract}
The object of the present paper is to study the transient magneto-thermo-visco-elastic stresses in a non-homogeneous anisotropic solid under initial stress. The system of fundamental equations is solved by means of a dual reciprocity boundary element method (DRBEM). In the case of plane deformation, a numerical scheme for the implementation of the method is presented and the numerical computations are presented graphically to show the effects of initial stress and inhomogeneity on the displacement components and thermal stress components.
\end{abstract}

Keywords: Magneto-Thermoviscoelastic Stresses, Initial Stress, Inhomogeneity, Anisotropic, Dual Reciprocity Boundary Element Method

\section{Introduction}

An increasing attention is being devoted to the interaction between magnetic field and strain field in an initially stressed anisotropic viscoelastic solid due to its many applications in modern aeronautics, astronautics, earthquake engineering, soil dynamics, nuclear reactors and high-energy particle accelerators. In recent years, an important number of engineering and mathematical papers devoted to the numerical solution have studied the overall behavior of such materials. El-Naggar, et al. [1,2] proposed explicit finite difference scheme to obtain thermal stresses in a non-homogeneous media. The boundary element method is well known for its accuracy and efficiency in stress analysis (see, for example, Brebbia and Nardini [3], Wrobel and Brebbia [4], Partridge, et al. [5], Divo and Kassab [6], Gaul, et al. [7], Matsumoto, et al. [8], Fahmy [9-11], Davi and Milazzo [12]).

The idea of the present paper is to study the transient magneto-thermo-visco-elastic stresses in a non-homogeneous anisotropic initially stressed solid. The formulation is tested through its application to the problem of a solid placed in a constant primary magnetic field acting in the direction of the z-axis. The governing equations are solved by means of a dual reciprocity boundary element method (DRBEM) and then numerical calculations are made for the temperature, displacement components and thermal stress components. The validity of DRBEM is examined by considering a magneto-thermo-viscoelastic solid occupies a rectangular region and good agreement is obtained with existent results. The results indicate that the effects of initial stress and inhomogeneity are very pronounced.

\section{Formulation of the Problem}

Here, we present the basic equations of the theory of magneto-thermoviscoelasticity, which will be used for the solution of the problem described above. With reference to a Cartesian frame denoted by 0xyz, consider an initially stressed anisotropic solid is placed in a constant primary magnetic field $H_{0}$, acting in the direction of the $z$-axis. Here we address the generalized two-dimensional deformation problem in $x y$-plane only, therefore all the variables are constant along the $\mathrm{z}$-axis. In the $0 x y$ plane, the solid occupies the region

$R=\{(x, y): 0<x<1,0<y<1\}$ which is bounded by a simple closed curve $C$.

In non-dimensional form the governing equations of magneto-thermo-viscoelasticity for an anisotropic solid can be written as follows (see Fahmy and El-Shahat [13]) 


$$
\begin{gathered}
\sigma_{p j, j}+\tau_{p j, j}-\Gamma_{p j}=\rho^{\prime} \ddot{u}_{p} \\
\sigma_{p j}=C_{p j k l}^{\prime} \aleph u_{k, l}-\beta_{p j}^{\prime} T(x, y, \tau) \\
\tau_{p j}=\mu^{\prime}\left(h_{p} H_{j}+h_{j} H_{p}-\delta_{j p}\left(h_{k} H_{k}\right)\right), \\
h_{p}=(\nabla \times(\mathbf{u} \times \mathbf{H}))_{p} \\
\Gamma_{p j}=\mathrm{P}^{\prime}\left(\frac{\partial u_{j}}{\partial x_{p}}-\frac{\partial u_{p}}{\partial x_{j}}\right) \\
k_{p j}^{\prime} T_{, p j}=c \rho^{\prime} \dot{T}-\rho^{\prime} r
\end{gathered}
$$

The initial and boundary conditions for the current problem are assumed to be written as

$$
\begin{gathered}
u_{k}(x, y, 0)=\dot{u}_{k}(x, y, 0)=0 \text { for }(x, y) \in R \cup C \\
u_{k}(x, y, \tau)=\Psi_{\mathrm{k}}(x, y, \tau) \text { for }(x, y) \in C_{3} \\
t_{k}(x, y, \tau)=\delta_{k}(x, y, \tau) \text { for }(x, y) \in C_{4} \\
T(x, y, 0)=f(x, y) \text { for }(x, y) \in R \cup C \\
T(x, y, \tau)=\mathrm{H}(x, y, \tau) \text { for }(x, y) \in C_{1}, \tau>0 \\
q(x, y, \tau)=\bar{h}(x, y, \tau) \text { for }(x, y) \in C_{2}, \tau>0
\end{gathered}
$$

where $x_{1}$ and $x_{2}$ are the same expressions $x$ and $y$ respectively, $\sigma_{p j}$ is the mechanical stress tensor, $\tau_{p j}$ Maxwell's electromagnetic stress tensor, $u_{k}$ is the displacement, $\mathrm{T}$ is the temperature, $P^{\prime}$ is the initial stress in the solid, $C_{p j k l}^{\prime}\left(C_{p j k l}^{\prime}=C_{k l p j}^{\prime}=C_{j p k l}^{\prime}\right)$ and $\beta_{p j}^{\prime}\left(\beta_{p j}^{\prime}=\beta_{j p}^{\prime}\right)^{p j k l}$ are respectively, the constant elastic moduli and stress-temperature coefficients of the anisotropic medium, $\aleph$ is the viscoelastic material constant, $\mu^{\prime}$ is the magnetic permeability, $h$ is the perturbed magnetic field, $k_{p j}^{\prime}$ are the thermal conductivity coefficients satisfying the symmetry relation $k_{p j}^{\prime}=k_{j p}^{\prime}$ and the strict inequality $\left(k_{12}^{\prime}\right)^{2}-k_{11}^{\prime} k_{22}^{\prime}<0$ holds at all points in the medium, $\rho^{\prime}$ is the density, $c$ is the specific heat capacity of the solid and $\tau$ is the dimensionless time, $\rho^{\prime} r$ is the heat source density and $r$ is the Euclidean distance between the field point $x$ and the load point $\xi$. Also, $\Psi_{\mathrm{k}}, \delta_{k}, \mathrm{H}$ and $\bar{h}$ are suitably prescribed functions of $(x, y, \tau), f$ is suitably prescribed function of $(x, y), C_{1}$ and $C_{2}$ are nonintersecting curves such that $C=C_{1} \cup C_{2}, C_{3}$ and $C_{4}$ are non-intersecting curves such that $C=C_{3} \cup C_{4}$ and $t_{k}$ are the tractions defined by $t_{k}=\sigma_{k j} n_{j}$.

A superposed dot denotes differentiation with respect to the time and a comma followed by a subscript denotes partial differentiation with respect to the corresponding coordinates.

We focus our attention to the case of inhomogeneity along $x$-axis, so we characterize the elastic constants $C_{p j k l}^{\prime}$, magnetic permeability $\mu^{\prime}$, initial stress $\mathrm{P}^{\prime}$, ther- mal conductivity coefficients $k_{p j}^{\prime}$ and density $\rho^{\prime}$ of non-homogeneous material by

$$
\begin{gathered}
C_{p j k l}^{\prime}=C_{p j k l}(x+1)^{m}, \beta_{p j}^{\prime}=\beta_{p j}(x+1)^{m}, \\
\mu^{\prime}=\mu(x+1)^{m}, \mathrm{P}^{\prime}=\mathrm{P}(x+1)^{m} \\
k_{p j}^{\prime}=k_{p j}(x+1)^{m}, \rho^{\prime}=\rho(x+1)^{m} .
\end{gathered}
$$

where $C_{p j k l}, \mu, \mathrm{P}, k_{p j}$ and $\rho$ are constants (the values of $C_{p j k l}^{\prime}, \mu^{\prime}, \mathrm{P}^{\prime}, k_{p j}^{\prime}$ and $\rho^{\prime}$ in homogeneous matter) and $m$ is a rational numbers.

In terms of the definitions (12) and (13), Equations (1) and (5) can be written as follows

$$
\begin{gathered}
\sigma_{p j, j}+\tau_{p j, j}-\mathrm{P}(x+1)^{m}\left(\frac{\partial u_{j}}{\partial x_{p}}-\frac{\partial u_{p}}{\partial x_{j}}\right) \\
-\rho(x+1)^{m} \omega^{2} x_{p}=\rho(x+1)^{m} \ddot{u}_{p} \\
k_{p j} T_{, p j}=c \rho \dot{T}-\rho r
\end{gathered}
$$

With the heat flux vector $q_{p}$ given by Fourier's law

$$
q_{p}=-k_{p j} T_{, j}
$$

where $k_{p j}$ and $c$ are taken to be constant in $R$.

It is usually difficult to find out the analytical solutions of such state equations except for some special cases.

\section{Numerical Implementation}

The main objective of the numerical implementation is to describe the implementation of the DRBEM formulation for the solution of the Equations (14) and (15). A more extensive historical review and applications of dual reciprocity boundary element method may be found in Brebbia, et al. [14], Partridge and Wrobel [15], Nardini and Brebbia [16], Albuquerque, et al. [17].

\subsection{Temperature Field}

Weighting (15) with a test function $T^{*}$ yields

$$
\int_{\mathrm{R}} k_{p j} T_{, p j} T^{*} \mathrm{dR}=\int_{\mathrm{R}}(c \rho \dot{T}-\rho r) T^{*} \mathrm{dR}
$$

Applying Gauss's theorem, integration by parts, Green's second identity and sifting property of the Dirac distribution, we obtain the representation formula

$$
T(\xi)=\int_{\mathrm{C}}\left(q^{*} T-T^{*} q\right) d \mathrm{C}-\int_{\mathrm{R}}(c \rho \dot{T}-\rho r) T^{*} \mathrm{dR}
$$

According to the DRBEM, the surface of the solid has to be discretized into boundary elements. In order to make the implementation easy to compute, we use $N_{b}$ collocation points on the boundary $C$ and another $N_{i}$ in the interior of $R$ so that the total number of interpolation points is $N=N_{b}+N_{i}$. 
According to Cho, et al. [18], a function of the form

$$
f=\sum_{k=1}^{N} a_{j} \Phi\left(\left\|x-x_{k}\right\|\right)+p_{w}(x)
$$

where $\Phi$ is a continuous function in the $x y$-plane and $p_{w}$ is a polynomial of degree $w$ is called a radial basis function $(\mathrm{RBF})$. The importance of splines is that they can provide interpolatory approximations to $f$ for very general sets of interpolation points $\left\{x_{k}\right\}_{k=1}^{N}$ in the $x y$ plane.

In the $x y$-plane a spline is of the form

$$
\Phi^{[n]}=r^{2 n} \log (r), n \geq 1
$$

where $r=\|x-\xi\|$ is the Euclidean distance between the field point $x$ and the load point $\xi$. and $p_{w}$ is a polynomial of degree $w=n$. For $n=1$ these are the thin plate splines (TPS)

$$
\Phi^{[1]}=r^{2} \log (r) \text { and } p_{1}=a x+b y+c
$$

Thus, the particular solution of the temperature can be approximated as

$$
T^{q}=\sum_{k=1}^{N} a_{k} \Psi_{k}(x)+\mathrm{X}_{w}(x)
$$

where

$$
\Delta \Psi_{k}-\lambda^{2} \Psi_{k}=\Phi_{k}(x), \Phi_{k}(x)=\Phi\left(x-x_{k}\right)
$$

and

$$
\Delta \mathrm{X}_{w}-\lambda^{2} \mathrm{X}_{w}=p_{w}
$$

Consequently, the dual reciprocity representation formula can be written as follows

$$
\begin{aligned}
& T(\xi)+\int_{\mathrm{C}}\left(T^{*} q-q^{*} T\right) \mathrm{dC} \\
& =\sum_{\mathrm{q}=1}^{\mathrm{N}}\left(T^{q}(\xi)+\int_{\mathrm{C}}\left(T^{*} q^{q}-q^{*} T^{q}\right) \mathrm{dC}\right) \bar{\alpha}^{q}
\end{aligned}
$$

In which all domain integrals have been transformed to the boundary.

The field variables $T$ and $q$ are then approximated by means of shape functions $\bar{\varphi}_{k}$ and nodal values $\breve{T}_{k}$ and $\breve{q}_{k}$ as follows

$$
\{T, q\} \approx \sum_{k=1}^{N} \bar{\varphi}_{k}\left\{\breve{T}_{k}, \breve{q}_{k}\right\}=\bar{\Phi}^{T}\{\breve{T}, \breve{q}\}
$$

where $\breve{T}, \breve{q}$ and $\bar{\Phi}^{T}$ are matrices

We can also approximate the particular solutions $T^{q}$ and $q^{q}$ on the boundary as the unknown field variables by means of nodal values $\breve{T}_{k}^{q}$ and $\breve{T}_{k}^{q}$ as follows

$$
\left\{T^{q}, q^{q}\right\} \approx \sum_{k=1}^{N} \bar{\varphi}_{k}\left\{\breve{T}_{k}^{q}, \breve{q}_{k}^{q}\right\}=\bar{\Phi}^{T}\left\{\breve{T}^{q}, \breve{q}^{q}\right\}
$$

where $\breve{T}^{q}$ and $\breve{q}^{q}$ are matrices
Using (22) and (23) and applying the point collocation procedure to (21), we have the following system of equations

$$
\bar{\zeta} \breve{T}-\bar{\eta} \breve{q}=\sum_{q=1}^{N}\left(\bar{\zeta} \breve{T}^{q}-\bar{\eta} \breve{q}^{q}\right) \bar{\alpha}^{q}
$$

Let

$$
\begin{aligned}
& \breve{\mathbf{T}}=\left[\begin{array}{llll}
\breve{T}^{1} & \breve{T}^{2} & \cdots & \breve{T}^{N}
\end{array}\right], \bar{\wp}=\left[\begin{array}{llll}
\breve{q}^{1} & \breve{q}^{2} & \cdots & \breve{q}^{N}
\end{array}\right], \\
& \bar{\alpha}=\left[\begin{array}{llll}
\bar{\alpha}^{1} & \bar{\alpha}^{2} & \cdots & \bar{\alpha}^{N}
\end{array}\right]^{T}
\end{aligned}
$$

Using (25) into (24) we have

$$
\bar{\zeta} \breve{T}-\bar{\eta} \breve{q}=(\bar{\zeta} \breve{\mathbf{T}}-\bar{\eta} \breve{\wp}) \bar{\alpha}
$$

where the matrices $\breve{\mathbf{T}}$ and $\breve{\varsigma}$ contain the particular solutions.

The generalized source term in (18) is approximated with a series of given source source terms $f^{q}$ and unknown coefficients $\bar{\alpha}^{q}$ as follows

$$
c \rho \dot{T}-\rho r \approx \sum_{q=1}^{N} f^{q} \bar{\alpha}^{q}
$$

Then, by applying a point collocation procedure to Equation (27) we obtain

$$
c \rho \grave{T}-\rho \breve{r}=\bar{F} \bar{\alpha}(\tau)
$$

Which can be substituted into (27) producing

$$
\overline{\mathcal{M}} \grave{T}(\tau)+\bar{\zeta} \breve{T}(\tau)=\bar{\eta} \breve{q}(\tau)+\breve{\mathrm{B}}(\tau)
$$

where

$$
\overline{\mathcal{M}}=c \rho v, \breve{\mathrm{B}}=\rho v \breve{Q}, v=-(\bar{\zeta} \breve{T}-\bar{\eta} \breve{\wp}) \bar{F}^{-1}
$$

In order to solve the system (29), the nodal vectors are subdivided into known and unknown parts denoted by the superscripts $k$ and $u$

$$
\left\{\breve{T}^{k}, \breve{q}^{u}\right\} \in \mathrm{C}_{1},\left\{\breve{T}^{u}, \breve{q}^{k}\right\} \in \mathrm{C}_{2}
$$

The following matrix equation is obtained from (29):

$$
\begin{aligned}
& {\left[\begin{array}{ll}
\overline{\mathcal{M}}^{11} & \overline{\mathcal{M}}^{12} \\
\overline{\mathcal{M}}^{21} & \overline{\mathcal{M}}^{22}
\end{array}\right]\left[\begin{array}{l}
\check{T}^{k}(\tau) \\
\dot{\bar{T}}^{u}(\tau)
\end{array}\right]+\left[\begin{array}{ll}
\bar{\zeta}^{11} & \bar{\zeta}^{12} \\
\bar{\zeta}^{21} & \bar{\zeta}^{22}
\end{array}\right]\left[\begin{array}{l}
\breve{T}^{k}(\tau) \\
\breve{T}^{u}(\tau)
\end{array}\right]} \\
& =\left[\begin{array}{ll}
\bar{\eta}^{11} & \bar{\eta}^{12} \\
\bar{\eta}^{21} & \bar{\eta}^{22}
\end{array}\right]\left[\begin{array}{l}
\bar{q}^{k}(\tau) \\
\bar{q}^{u}(\tau)
\end{array}\right]+\left[\begin{array}{c}
\breve{B}^{1}(\tau) \\
\breve{B}^{2}(\tau)
\end{array}\right]
\end{aligned}
$$

The unknown fluxes $\breve{q}^{u}(t)$ are obtained from the first row of matrix Equation (32) are expressed as follows

$$
\begin{aligned}
\breve{q}^{u}(\tau)= & \left(\bar{\eta}^{12}\right)^{-1}\left[\overline{\mathcal{M}}^{11} \breve{\dot{T}}^{k}(\tau)+\overline{\mathcal{M}}^{12} \breve{\dot{T}}^{u}(\tau)+\bar{\zeta}^{11} \breve{T}^{k}(\tau)\right. \\
& \left.+\bar{\zeta}^{12} \breve{T}^{u}(\tau)-\bar{\eta}^{11} \breve{q}^{k}(\tau)-\breve{B}^{1}(\tau)\right]
\end{aligned}
$$


Making use of (33) we can write the second row of matrix Equation (32) as follows

$$
\overline{\mathcal{M}}^{u} \check{\dot{T}}^{u}(\tau)+\bar{\zeta}^{u} \breve{T}^{u}(\tau)=\breve{Q}^{k}(\tau)
$$

where

$$
\begin{aligned}
\bar{Q}^{k}(\tau)=\breve{B}^{k}(\tau)+\bar{\eta}^{k} \breve{q}^{k}(\tau)-\overline{\mathcal{M}}^{k} \breve{\dot{T}}^{k}(\tau)-\bar{\zeta}^{k} \breve{T}^{k}(\tau), \\
\overline{\mathcal{M}}^{u}=\overline{\mathcal{M}}^{22}-\bar{\eta}^{22}\left(\bar{\eta}^{22}\right)^{-1} \overline{\mathcal{M}}^{12}, \\
\bar{\zeta}^{u}=\bar{\zeta}^{22}-\bar{\eta}^{22}\left(\bar{\eta}^{22}\right)^{-1} \bar{\zeta}^{12}, \\
\breve{B}^{k}(\tau)=B^{2}(\tau)-\bar{\eta}^{22}\left(\bar{\eta}^{12}\right)^{-1} B^{1}(\tau), \\
\bar{\eta}^{k}=\bar{\eta}^{21}-\bar{\eta}^{22}\left(\bar{\eta}^{12}\right)^{-1} \bar{\eta}^{11}, \\
\overline{\mathcal{M}}^{k}=\overline{\mathcal{M}}^{21}-\bar{\eta}^{22}\left(\bar{\eta}^{12}\right)^{-1} \overline{\mathcal{M}}^{11}, \\
\bar{\zeta}^{k}=\bar{\zeta}^{21}-\bar{\eta}^{22}\left(\bar{\eta}^{12}\right)^{-1} \bar{\zeta}^{11} .
\end{aligned}
$$

At a time step $n+1$, Equation (34) can be written in the following form

$$
\overline{\mathcal{M}}^{u} \stackrel{\grave{T}}{n+1}^{u}+\bar{\zeta}^{u} \breve{T}_{n+1}^{u}=\breve{Q}_{n+1}^{k}
$$

where

$$
Q_{n+1}^{k}(\tau)=\breve{B}_{n+1}^{k}(\tau)+\bar{\eta}^{k} \breve{q}_{n+1}^{k}(\tau)-\overline{\mathcal{M}}^{k}{\breve{T_{n+1}^{k}}}^{k}(\tau)-\bar{\zeta}^{k} \breve{T}_{n+1}^{k}(\tau)
$$

We take the finite difference grids with $\Delta \tau$ as the time step, and use the subscripts $n$ to denote the $n t h$ discrete time. A mesh is defined by

$\tau=\Delta \tau n, \tau=\Delta \tau n$ being the time step

Using finite difference, we can approximate the temperature as follows

$$
\breve{T}_{n+1}=\frac{\breve{T}_{n+1}-\breve{T}_{n}}{\Delta \tau}
$$

Hence, we can write

$$
\bar{\varsigma}^{u} \breve{T}_{n+1}^{u}=\overline{\mathbb{Q}}_{n+1}^{k}
$$

where

$$
\begin{gathered}
\overline{\mathbb{Q}}_{n+1}^{k}=\bar{Q}_{n+1}^{k}+\frac{\overline{\mathcal{M}}^{u}}{\Delta \tau} \breve{T}_{n}^{u} \\
\bar{\zeta}^{u}=\frac{\overline{\mathcal{M}}^{u}}{\Delta \tau}+\bar{\zeta}^{u}
\end{gathered}
$$

Thus, with $T(x, y, t)$ determined, the remaining task is to solve (14) subject to (6), (7) and (8).

\subsection{Displacement Field}

Making use of (2)-(4), (12) and (13), we can write (14) as follows

$$
\begin{aligned}
L_{p k} u_{k} & =\rho \ddot{u}_{p}-\left(\left(D_{p l}+\Lambda D_{p 1 k}\right) u_{k}+D_{p} T-\mathrm{P} D_{j p}\right) \\
& =\rho \ddot{u}_{p}-\rho b_{p}=\rho b_{p}^{\prime}
\end{aligned}
$$

where

$$
\begin{gathered}
L_{p k}=D_{p j k} \frac{\partial}{\partial x_{j}}, D_{p j k}=C_{p j k l} \aleph \frac{\partial}{\partial x_{l}}, \\
D_{p}=-\beta_{p j}\left(\frac{\partial}{\partial x_{j}}+\delta_{j 1} \Lambda\right), \Lambda=\frac{\mathrm{m}}{x+1}, \\
D_{p l}=\mu H_{0}^{2}\left(\frac{\partial}{\partial x_{p}}+\delta_{p 1} \Lambda\right) \frac{\partial}{\partial x_{l}}, D_{j p}=\left(\frac{\partial u_{j}}{\partial x_{p}}-\frac{\partial u_{p}}{\partial x_{j}}\right) .
\end{gathered}
$$

When the temperature field is known, the displacement field is obtained by solving (38), where the inertia term $\rho \ddot{u}_{p}$, the magnetic term $D_{p l} u_{k}$, the viscosity term $\Lambda D_{p 1 k} u_{k}$, the temperature gradient $D_{p} T$ and the initial stress term $\mathrm{P} D_{j p}$ are treated as the body forces.

On the basis of the method of weighted residuals, the differential Equation (38) can be transformed to the integral equation in the following form

$$
\int_{\mathrm{R}}\left(L_{p k} u_{k}-\rho b_{p}^{\prime}\right) u_{p}^{*} \mathrm{dR}=0
$$

where $u_{p}^{*}$ is a weighting function and $u_{k}$ is the approximate solution.

Integration by parts twice using the divergence theorem of Gauss as in Fahmy [11] yields

$$
\begin{aligned}
& \int_{\mathrm{R}}\left(L_{p k} u_{k} \cdot u_{p}^{*}-L_{p k}^{*} u_{k}^{*} \cdot u_{p}\right) \mathrm{dR} \\
& =\int_{\mathrm{C}}\left(G_{p k} u_{k} \cdot u_{p}^{*}-G_{p k}^{*} u_{k}^{*} \cdot u_{p}\right) \mathrm{dC}
\end{aligned}
$$

where the traction vectors on the boundary are

$$
\begin{aligned}
t_{p} & =C_{p j k l} \aleph u_{k, l} n_{j}=G_{p k} u_{k} \text { and } \\
t_{p}^{*} & =C_{p j k l} \aleph u_{k, j}^{*} n_{l}=G_{p k}^{*} u_{k}^{*}
\end{aligned}
$$

Using the symmetric elasticity tensor $\left(C_{p j k l}=C_{k j p l}\right)$ Therefore, it follows that

$$
\begin{gathered}
L_{p k}=C_{p j k l} \aleph \frac{\partial^{2}}{\partial x_{l} \partial x_{j}}=C_{k j p l} \aleph \frac{\partial^{2}}{\partial x_{j} \partial x_{l}}=L_{p k}^{*} \\
G_{p k}=C_{p j k l} \aleph n_{j} \frac{\partial}{\partial x_{l}}=C_{k j p l} \aleph n_{l} \frac{\partial}{\partial x_{j}}=G_{p k}^{*}
\end{gathered}
$$

Using Equations (41), (42) and (43), the Equation (40) can now be rewritten in the form

$$
\begin{aligned}
& \int_{\mathrm{R}}\left(C_{p j k l} \aleph u_{k, l j} \cdot u_{p}^{*}-C_{p j k l} \aleph u_{k, l j}^{*} \cdot u_{p}\right) \mathrm{dR} \\
& =\int_{\mathrm{C}}\left(t_{p} u_{p}^{*}-t_{p}^{*} u_{p}\right) \mathrm{dC}
\end{aligned}
$$

We define the fundamental solution $u_{m k}^{*}$ by the relation 


$$
L_{j l} u_{m k}^{*}=-\delta(x, \xi) \delta_{p m}
$$

If we replace the weighting functions $[\cdot]_{k}^{*}$ in (44) by $[\cdot]_{m k}^{*}$, then we have

$$
\begin{aligned}
& \int_{\mathrm{R}}\left(C_{p j k l} \aleph u_{k, l j} \cdot u_{m p}^{*}-C_{p j k l} \aleph u_{m k, l j}^{*} \cdot u_{p}\right) \mathrm{dR} \\
& =\int_{\mathrm{C}}\left(t_{p} u_{m p}^{*}-t_{m p}^{*} u_{p}\right) \mathrm{dC}
\end{aligned}
$$

From (38), (45) and (46), the representation formula may be written as

$$
\begin{aligned}
u_{m}(\xi)= & \int_{\mathrm{C}}\left(u_{m p}^{*}(x, \xi) t_{p}(x)-t_{m p}^{*}(x, \xi) u_{p}(x)\right) \mathrm{dC} \\
& -\int_{\mathrm{R}} u_{m p}^{*}(x, \xi) \rho b_{p}^{\prime}(x) \mathrm{d} \mathrm{R}
\end{aligned}
$$

Let

$$
\begin{aligned}
\rho b_{p}^{\prime} & =\rho \ddot{u}_{p}-\left(\left(D_{p l}+\Lambda D_{p 1 k}\right) u_{k}+D_{p} T-\mathrm{P} D_{j p}\right) \\
& \approx \sum_{\mathrm{q}=1}^{\mathrm{N}} f_{p n}^{q} \alpha_{n}^{q}=\sum_{\mathrm{q}=1}^{\mathrm{N}}\left(L_{p k} u_{k n}^{q}\right) \alpha_{n}^{q}
\end{aligned}
$$

Using the TPS as in Cho, et al. [18], we can write the particular solution of the displacement as follows

$$
u_{k n}^{q}=\left\{\begin{array}{l}
-\frac{4}{\lambda^{4}}\left[K_{0}(\lambda r)+\log (r)\right]-\frac{r^{2} \log r}{\lambda^{2}}-\frac{4}{\lambda^{4}}, r>0 \\
\frac{4}{\lambda^{4}}\left[\gamma+\log \left(\frac{\lambda}{2}\right)\right]-\frac{4}{\lambda^{4}}, r=0
\end{array}\right.
$$

where $K_{0}$ is the Bessel function of the third kind of order zero and $\gamma=0.5772156649015328$, which is known as Euler's constant.

Hence, the traction particular solution $t_{p n}^{q}$ and source function $f_{p n}^{q}$ can be obtained by evaluating

$$
t_{p n}^{q}=C_{p j k l} u_{k n, l}^{q} n_{j}, L_{p k} u_{k n}^{q}=f_{p n}^{q}
$$

On the basis of these considerations, the integral domain can be approximated as

$$
\int_{\mathrm{R}} u_{m p}^{*} \rho b_{p}^{\prime} \mathrm{dR} \approx \sum_{\mathrm{q}=1}^{\mathrm{N}}\left(\int_{\mathrm{R}}\left(L_{p k} u_{k n}^{q} u_{m p}^{*}\right) \mathrm{dR}\right) \alpha_{n}^{q}
$$

The use of (51) together with the dual reciprocity

$$
\begin{aligned}
& \int_{\mathrm{R}}\left(L_{p k} u_{k n}^{q} u_{m p}^{*}-L_{p k} u_{m k}^{*} u_{p n}^{q}\right) \mathrm{dR} \\
& =\int_{\mathrm{C}}\left(u_{m p}^{*} t_{p n}^{q}-t_{m p}^{*} u_{p n}^{q}\right) \mathrm{dC}
\end{aligned}
$$

Gives rise to

$$
\begin{aligned}
& \int_{\mathrm{R}} u_{m p}^{*} \rho b_{p}^{\prime} \mathrm{dR} \\
& =\sum_{\mathrm{q}=1}^{\mathrm{N}}\left(\int_{\mathrm{R}}\left(L_{p k} u_{m k}^{*} u_{p n}^{q}\right) \mathrm{dR}+\int_{\mathrm{C}}\left(u_{m p}^{*} t_{p n}^{q}-t_{m p}^{*} u_{p n}^{q}\right) \mathrm{dC}\right) \alpha_{n}^{q}
\end{aligned}
$$

From (45), one may derive

$$
\int_{\mathrm{R}} L_{j l} u_{m k}^{*} u_{p n}^{q} \mathrm{dR}=\int_{\mathrm{R}}-\delta(x, \xi) \delta_{p m} u_{p n}^{q} \mathrm{dR}=-u_{m n}^{q}(\xi)
$$

Making use of (47), (53) and (54) we can write the dual reciprocity representation formula as follows

$$
\begin{aligned}
u_{m}(\xi)= & \int_{\mathrm{C}}\left(u_{m p}^{*} t_{p}-t_{m p}^{*} u_{p}\right) \mathrm{dC} \\
& +\sum_{q=1}^{N}\left(u_{m n}^{q}(\xi)-\int_{\mathrm{C}}\left(u_{m p}^{*} t_{p n}^{q}-t_{m p}^{*} u_{p n}^{q}\right) \mathrm{dC}\right) \alpha_{\mathrm{n}}^{\mathrm{q}}
\end{aligned}
$$

The representation formula (55) is only valid if $\xi$ lies inside the domain $\mathrm{R}$. To obtain an expression that contains only boundary variables, the load point $\xi$ has to be moved to the boundary. Therefore, the boundary is deformed by a small circular region with radius $\varepsilon$ around the load point $\xi \in C$ as shown in Figure 1.

According to references [11], [19] and [20], the dual reciprocity boundary integral equation can be expressed as

$$
\begin{aligned}
& c_{p j} u_{p}(\varepsilon)+\int_{\mathrm{C}} u_{p} t_{m p}^{*} \mathrm{~d} \Gamma-\int_{\mathrm{C}} u_{m p}^{*} t_{p} \mathrm{~d} \Gamma \\
& =\sum_{\mathrm{q}=1}^{\mathrm{N}}\left(c_{p j} u_{p n}^{q}(\varepsilon)+\int_{\mathrm{C}} u_{p n}^{q} t_{m p}^{*} d \Gamma-\int_{\mathrm{C}} u_{m p}^{*} t_{p n}^{q} d \Gamma\right) \alpha_{\mathrm{n}}^{\mathrm{q}}
\end{aligned}
$$

where $\oint$ is the Cauchy principal value symbol.

The unknown field variables and the particular solutions are respectively approximated by means of nodal values $[\because]_{k}$ and shape functions $\varphi_{k}$

$$
\begin{gathered}
\{u, t\} \approx \sum_{k=1}^{N} \varphi_{k}\left\{\breve{u}_{k}, \breve{t}_{k}\right\}=\Phi^{T}\{\breve{u}, \breve{t}\} \\
\left\{u^{q}, t^{q}\right\} \approx \sum_{k=1}^{N} \varphi_{k}\left\{\breve{u}_{k}^{q}, \breve{t}_{k}^{q}\right\}=\Phi^{T}\left\{\breve{u}^{q}, \breve{t}^{q}\right\}
\end{gathered}
$$

where $\left[\ulcorner]_{k}\right.$ and $\Phi$ are matrices.

On the basis of these approximations, and using the point collocation procedure, the dual reciprocity boundary integral Equation (56) results to the following system of equations

$$
\zeta \breve{u}-\eta \breve{t}=\sum_{q=1}^{N}\left(\zeta \breve{u}^{q}-\eta \breve{t}^{q}\right) \alpha^{q}(\tau)
$$

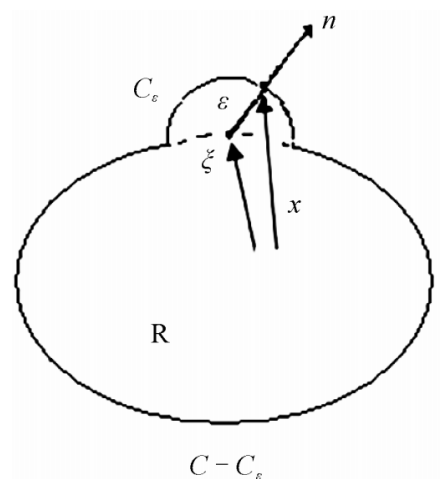

Figure 1. Geometry of the deformed boundary. 
By letting

$$
\begin{aligned}
\breve{U} & =\left[\begin{array}{llll}
\breve{u}^{1} & \breve{u}^{2} & \cdots & \breve{u}^{N}
\end{array}\right], \breve{\wp}=\left[\begin{array}{llll}
\breve{t}^{1} & \breve{t}^{2} & \cdots & \breve{t}^{N}
\end{array}\right], \\
\alpha & =\left[\begin{array}{llll}
\alpha^{1} & \alpha^{2} & \cdots & \alpha^{N}
\end{array}\right]^{T}
\end{aligned}
$$

We can write (59) as follows

$$
\zeta \breve{u}(\tau)-\eta \breve{t}(\tau)=(\zeta \breve{U}-\eta \breve{\wp}) \alpha(\tau)
$$

The coefficient vector $\alpha(\tau)$ can be calculated from (48) using the point collocation procedure, which yields

$$
\rho \breve{u}(\tau)-\rho \breve{b}(\tau)=F \alpha(\tau)
$$

Thus, (61) yields the system

$$
\mathcal{M} \breve{u}+\zeta \breve{u}=\eta \breve{t}(\tau)+\breve{\mathcal{B}}(\tau)
$$

where

$$
\square=(\eta \breve{\wp}-\zeta \breve{U}) F^{-1}, \mathcal{M}=\rho \square, \breve{\mathcal{B}}(\tau)=\rho \square \breve{b}(\tau) .
$$

By subdividing the nodal vectors into known and unknown parts as follows

$$
\left\{\breve{u}^{k}, \breve{t}^{u}\right\} \in \mathrm{C}_{3},\left\{\breve{u}^{u}, \breve{t}^{k}\right\} \in \mathrm{C}_{4}
$$

where the superscripts $k$ and $u$ denote, respectively, the known and unknown parts form

Hence we can write the system (63) in the following

$$
\begin{aligned}
& {\left[\begin{array}{ll}
\mathcal{M}^{11} & \mathcal{M}^{12} \\
\mathcal{M}^{21} & \mathcal{M}^{22}
\end{array}\right]\left[\begin{array}{l}
\ddot{u}^{k}(\tau) \\
\ddot{u}^{u}(\tau)
\end{array}\right]+\left[\begin{array}{ll}
\zeta^{11} & \zeta^{12} \\
\zeta^{21} & \zeta^{22}
\end{array}\right]\left[\begin{array}{l}
\breve{u}^{k}(\tau) \\
\breve{u}^{u}(\tau)
\end{array}\right]} \\
& =\left[\begin{array}{ll}
\eta^{11} & \eta^{12} \\
\eta^{21} & \eta^{22}
\end{array}\right]\left[\begin{array}{l}
\breve{t}^{k}(\tau) \\
\breve{t}^{u}(\tau)
\end{array}\right]+\left[\begin{array}{l}
\breve{\mathcal{B}}^{1}(\tau) \\
\breve{\mathcal{B}}^{2}(\tau)
\end{array}\right]
\end{aligned}
$$

The unknown fluxes $\breve{t}^{u}(\tau)$ can be obtained from the first row of (66) as follows

$$
\begin{aligned}
\breve{t}^{u}(\tau)= & \left(\eta^{12}\right)^{-1}\left[\mathcal{M}^{11} \breve{u}^{k}(\tau)+\mathcal{M}^{12} \breve{u}^{u}(\tau)+\zeta^{11} \breve{u}^{k}(\tau)\right. \\
& \left.+\zeta^{12} \breve{u}^{u}(\tau)-\eta^{11} \breve{t}^{k}(\tau)-\breve{\mathcal{B}}^{1}(\tau)\right]
\end{aligned}
$$

With the aid of (67) into the second row of (66) we obtain

$$
\mathcal{M}^{u} \breve{u}^{u}(\tau)+\zeta^{u} \breve{u}^{u}(\tau)=Q^{k}(\tau)
$$

where

$$
\begin{aligned}
Q^{k}(\tau)=\breve{\mathcal{B}}^{k}(\tau) & +\eta^{k} \breve{t}^{k}(\tau)-\mathcal{M}^{k} \breve{t}^{k}(\tau)-\zeta^{k} \breve{u}^{k}(\tau) \\
\mathcal{M}^{u}= & \mathcal{M}^{22}-\eta^{22}\left(\eta^{12}\right)^{-1} \mathcal{M}^{12} \\
\zeta^{u} & =\zeta^{22}-\eta^{22}\left(\eta^{12}\right)^{-1} \zeta^{12} \\
\mathcal{M}^{k} & =\mathcal{M}^{21}-\eta^{22}\left(\eta^{12}\right)^{-1} \mathcal{M}^{11}
\end{aligned}
$$

$$
\begin{gathered}
\zeta^{k}=\zeta^{21}-\eta^{22}\left(\eta^{12}\right)^{-1} \zeta^{11} \\
\breve{\mathcal{B}}^{k}(\tau)=\mathcal{B}^{2}(\tau)-\eta^{22}\left(\eta^{12}\right)^{-1} \mathcal{B}^{1}(\tau)
\end{gathered}
$$

We can write (68) at time step $n+1$

$$
\mathcal{M}^{u} \breve{u}_{n+1}^{u}(\tau)+\zeta^{u} \breve{u}_{n+1}^{u}(\tau)=Q_{n+1}^{k}(\tau)
$$

where

$$
Q_{n+1}^{k}(\tau)=\breve{\mathcal{B}}_{n+1}^{k}(\tau)+\eta^{k} \breve{t}_{n+1}^{k}(\tau)-\mathcal{M}^{k} \ddot{u}_{n+1}^{k}(\tau)-\zeta^{k} \breve{u}_{n+1}^{k}(\tau)
$$

Now, we consider an implicit backward finite difference scheme for solving the system of ordinary differential Equation (69), the so-called Houbolt's algorithm is applied to reduce (69) to an algebraic system. To do this, the velocities $\dot{u}_{n+1}$ and accelerations $\dot{u}_{n+1}$ at time step $n+1$ are approximated as follows

$$
\begin{gathered}
\breve{\ddot{u}}_{n+1} \approx \frac{1}{6 \Delta t}\left(11 \breve{u}_{n+1}-18 \breve{u}_{n}+9 \breve{u}_{n-1}-2 \breve{u}_{n-2}\right) \\
\breve{u}_{n+1} \approx \frac{1}{6 \Delta t}\left(11 \breve{u}_{n+1}-18 \breve{u}_{n}+9 \breve{u}_{n-1}-2 \breve{u}_{n-2}\right)
\end{gathered}
$$

Substituting (94) and (95) into (93) we have

$$
\varsigma^{u} \breve{u}_{n+1}^{u}(\tau)=\mathbb{Q}_{n+1}^{k}(\tau)
$$

where

$$
\begin{gathered}
\varsigma^{u}=\frac{2 \mathcal{M}^{u}}{\Delta t^{2}}+\zeta^{u} \\
\mathbb{Q}_{n+1}^{k}=Q_{n+1}^{k}+\frac{\mathcal{M}^{u}}{\Delta t^{2}}\left(5 \breve{u}_{n}-4 \breve{u}_{n-1}+\breve{u}_{n-2}\right)
\end{gathered}
$$

We apply successive over-relaxation (SOR) as described in Golub and Van Loan [21] to solve the system (96). For every time step $n+1$ the values of $\breve{u}_{n+1}^{u}$ are established. Once these values have been obtained, the unknown $\dot{\ddot{u}}_{n+1}^{u}$ and $\breve{u}_{n+1}^{u}$ can be obtained from (70) and (71), respectively. For the case in which $n=0$, the procedure described in Bathe [22] is used together with the initial conditions to derive $u_{-1}$ and $u_{-2}$. Lastly, we compute the traction vector $t_{n+1}^{u}$ using (67).

\section{Numerical Results and Discussion}

The present work should be applicable to any magneto-thermo-viscoelastic deformation problem. The application is for purpose of illustration; we do not intend to validate the results in a quantitative way because we have no experimental data at hand; this may be justified because our objective is to introduce a viable numerical technique for studying a model rather than to study any physical behaviors of it (see, for example, Ahmed [23], Kanaun [24] and Monsia [25]). Such a technique was 
discussed in Abd-Alla et al. [26-28] who solved the special case from this study in the absence of viscosity and inertia. To achieve better efficiency than the technique described in Abd-Alla, et al. [26-28], we use thin plate splines into a code, which is proposed in the current study. We extend the study of Abd-Alla, et al. [26-28], to include the viscosity interactions and the inertia term. Thus, it is perhaps not surprising that the numerical values obtained here are in very good agreement with those obtained by Abd-Alla et al. [26-28].

The example considered by Sladek, et al. [29] may be considered as a special case of the current general problem in the context of the uncoupled thermoelasticity theory. Also, there are alot of practical applications may be deduced as special cases from this general study and may be implemented in commercial finite element method (FEM) software packages FlexPDE 6.

In the special case under consideration, the results of the displacement $u_{1}$ are plotted in Figure 8 to show the validity of the proposed method. These results obtained with the DRBEM have been compared graphically with those obtained using the Meshless Local Petrov-Galerkin (MLPG) method of Sladek, et al. [29] and also the results obtained from the FlexPDE 6 are shown graphically in the same figures to confirm the validity of the proposed method. It can be seen from this figure that the DRBEM results are in excellent agreement with the results obtained by MLPG and finite element methods, thus confirming the accuracy of the DRBEM.With a view to illustrate the numerical implementation presented earlier, we consider a monoclinic graphite- epoxy as an anisotropic magneto-thermo-visco-elastic solid with the following physical constants:

Elasticity tensor

$$
C_{p j k l}=\left[\begin{array}{ccccccc}
430.1 & 130.4 & 18.2 & 0 & 0 & 201.3 \\
130.4 & 116.7 & 21.0 & 0 & 0 & 70.1 \\
18.2 & 21.0 & 73.6 & 0 & 0 & 2.4 \\
0 & 0 & 0 & 19.8 & -8.0 & 0 \\
0 & 0 & 0 & -8.0 & 29.1 & 0 \\
201.3 & 70.1 & 2.4 & 0 & 0 & 147.3
\end{array}\right] \mathrm{GPa}
$$

Mechanical temperature coefficient

$$
\beta_{p j}=\left[\begin{array}{ccc}
1.01 & 2.00 & 0 \\
2.00 & 1.48 & 0 \\
0 & 0 & 7.52
\end{array}\right] \cdot 10^{6} \mathrm{~N} / \mathrm{Km}^{2}
$$

Tensor of thermal conductivity

$$
k_{p j}=\left[\begin{array}{ccc}
5.2 & 0 & 0 \\
0 & 7.6 & 0 \\
0 & 0 & 38.3
\end{array}\right] \mathrm{W} / \mathrm{Km}
$$

Mass density $\rho=7820 \mathrm{~kg} / \mathrm{m}^{3}$ and heat capacity $c=461 \mathrm{~J} /(\mathrm{kg} \mathrm{K}), H_{0}=1000000$ Oersted, $\mu=0.5$ Gauss/Oersted, $\tau_{0}=0.5, m=0.5, \Delta \tau=0.0001$. The boundary $C_{1}$ is $x=0$, the boundary $C_{3}$ is $x=0$, the boundary $C_{2}$ is $x=1$, while $C_{4}$ is $x=1$. The numerical values of the temperature and displacement are obtained by discretizing the boundary into 120 elements $\left(N_{b}=120\right)$ and choosing 60 well spaced out collocation points $\left(N_{i}=60\right)$ in the interior of the solution domain.

The initial and boundary conditions considered in the calculations are

$$
\begin{gathered}
\text { at } \tau=0 u_{1}=u_{2}=\dot{u}_{1}=\dot{u}_{2}=0, T=1 \\
\text { at } x=0 u_{1}=u_{2}=0, T=0 \\
\text { at } x=1 \frac{\partial u_{1}}{\partial x}=\frac{\partial u_{2}}{\partial x}=0, \frac{\partial T}{\partial x}=0 \\
\text { at } y=0 u_{1}=u_{2}=0, T=0 \\
\text { at } y=1 \frac{\partial u_{1}}{\partial y}=\frac{\partial u_{2}}{\partial y}=0, \frac{\partial T}{\partial y}=0
\end{gathered}
$$

In order to evaluate the influence of the inhomogeneity on the displacements and thermal stresses in an anisotropic viscoelastic solid under initial stress, the inhomogeneity parameter is taken to be $m=0.5$ and for the homogeneous solid, we assume that $m=0.0$.

A comparison of the results is presented graphically for the following different cases: the solid line denoted by " $A$ " represents the solution for homogeneous solid in the absence of initial stress $(P=0)$, the dashed line denoted by " $\mathrm{C}$ " represents the solution for non-homogeneous solid in the absence of initial stress, the dotted line denoted by "B" represents the solution for homogeneous solid in the presence of initial stress $(\mathrm{P}=0.5)$ and the dashed-dotted line denoted by "D" represents the solution for non-homogeneous solid in the presence of initial stress.

Figure 2 shows the variation of the temperature $\mathrm{T}$ along $x$-axis at various values of the time $\tau$. It is noticed that the temperature increases with the increase of $x$ and $\tau$.

Figures 3-7 show the influence of the initial stress and inhomogeneity of the material constants on the displacements $u_{1}$ and $u_{2}$ and thermal stresses $\sigma_{11}, \sigma_{12}$ and $\sigma_{22}$. Also, they show the difference among the four cases "A", "C", "B" and "D".

Figure 3 shows that the displacement $u_{1}$ increases and then decreases with the increase of $x$. It is noticed that the maximum value happens in the homogeneous solid in the presence of initial stress.

Figure 4 shows that the displacement $u_{2}$ decreases and then increases for all cases. It is clear from this figure that the homogeneous and non-homogeneous curves 


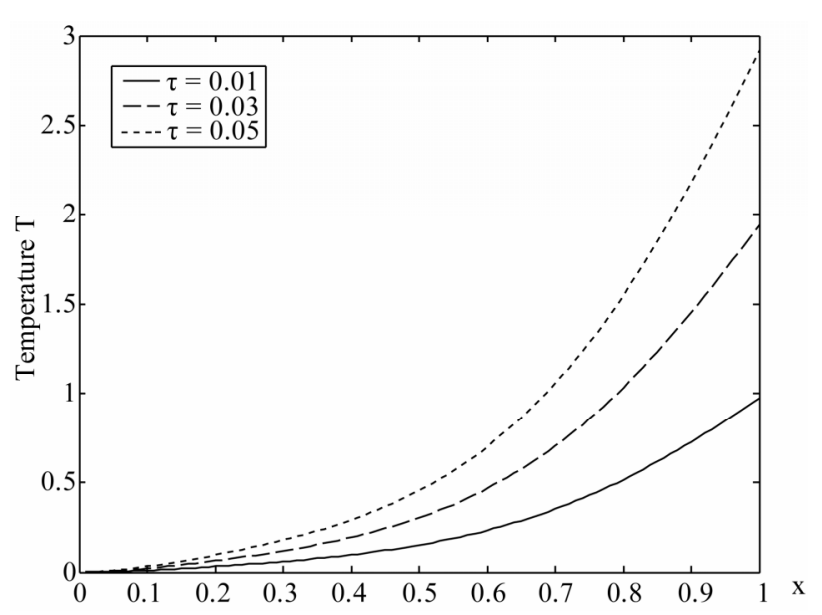

Figure 2. Variation of the temperature $T$ with $x$ coordinate $(\mathbf{y}=1)$.

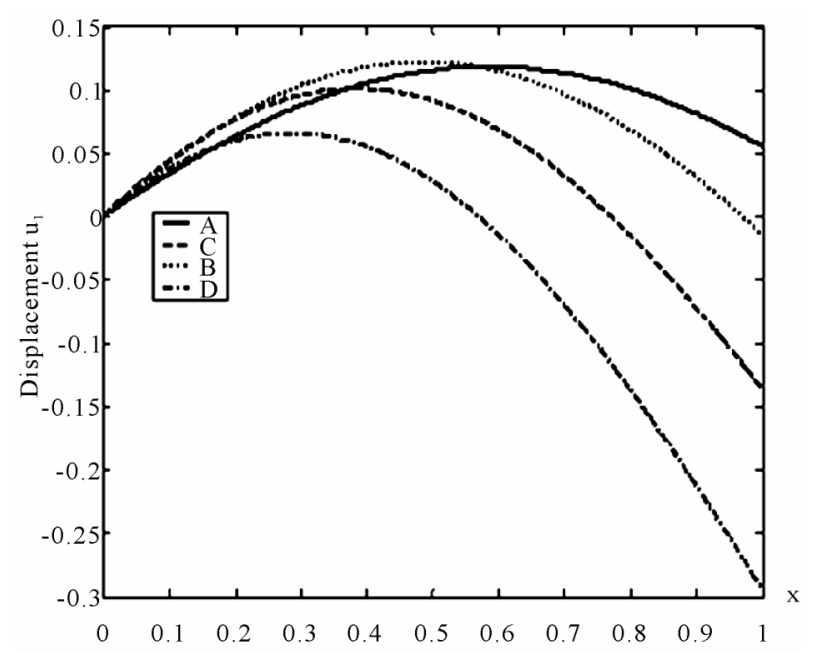

Figure 3. Variation of the displacement $u_{1}$ with $x$ coordinate.

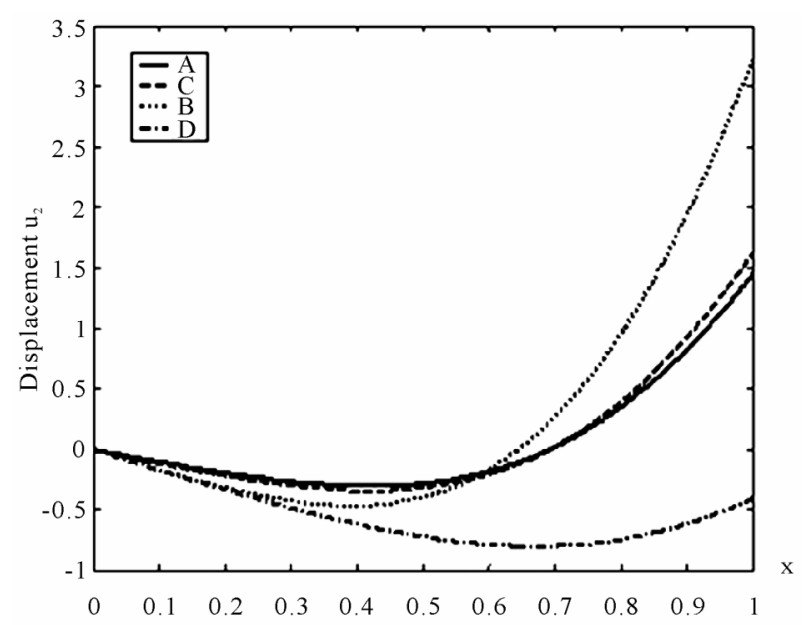

Figure 4. Variation of the displacement $u_{2}$ with $x$ coordinate. diverge in the presence of initial stress. We can see also from this figure that the negative maximum value happens in the non-homogeneous solid in the presence of initial stress.

Figure 5 shows that the thermal stress $\sigma_{11}$ increases with increasing $x$ for all cases. It is apparent from this figure that the increasing rate is more pronounced in non-homogeneous solid in the presence of initial stress.

Figure 6 shows that the thermal stress $\sigma_{12}$ increases with the increase of $x$ for all cases. It will be observed from this figure that the increasing rate is more pronounced in the presence of initial stress.

Figure 7 shows that the thermal stress $\sigma_{22}$ decreases with the increase of $x$ for the non-homogeneous solid, but for homogeneous solid it increases with the increase of $x$. It is clear from this figure that the increasing rate is more pronounced in the presence of initial stress.

It is clear from all of these figures that the curves of the displacements $u_{1}$ and $u_{2}$ and thermal stresses $\sigma_{11}$,

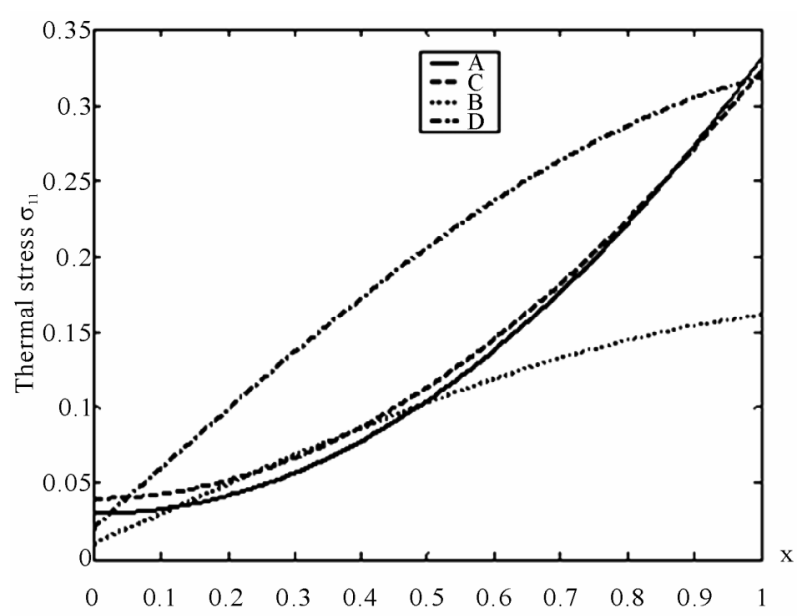

Figure 5. Variation of the thermal $\sigma_{11}$ with $x$ coordinate.

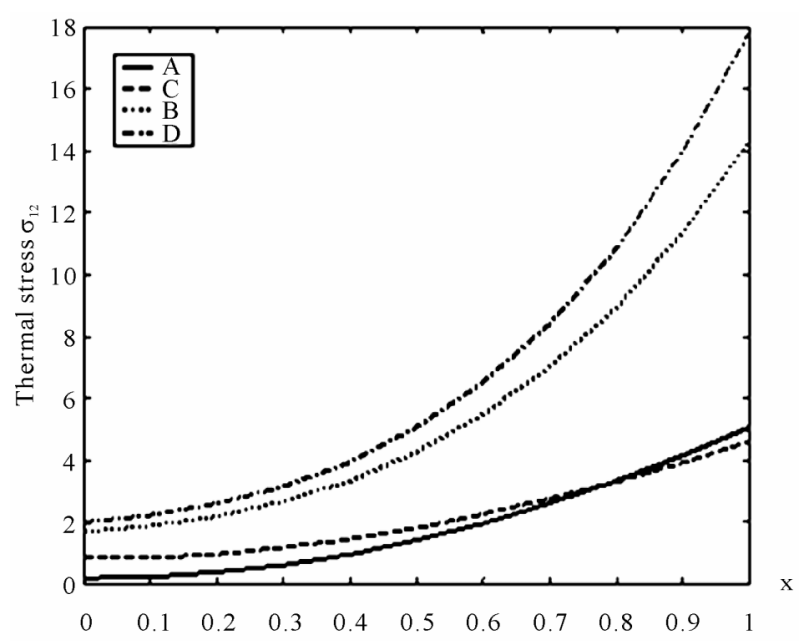

Figure 6. Variation of the thermal $\sigma_{12}$ with $\mathrm{x}$ coordinate. 


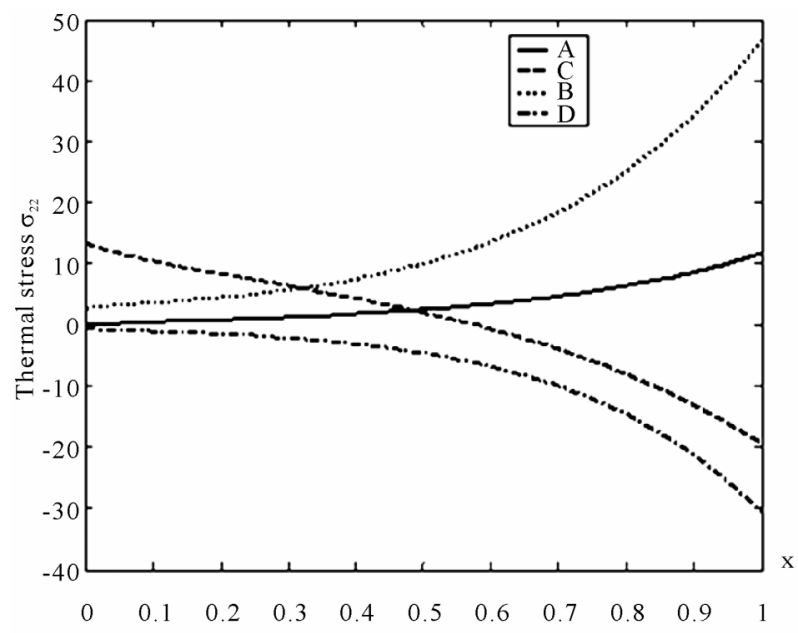

Figure 7. Variation of the thermal $\sigma_{22}$ with $x$ coordinate.

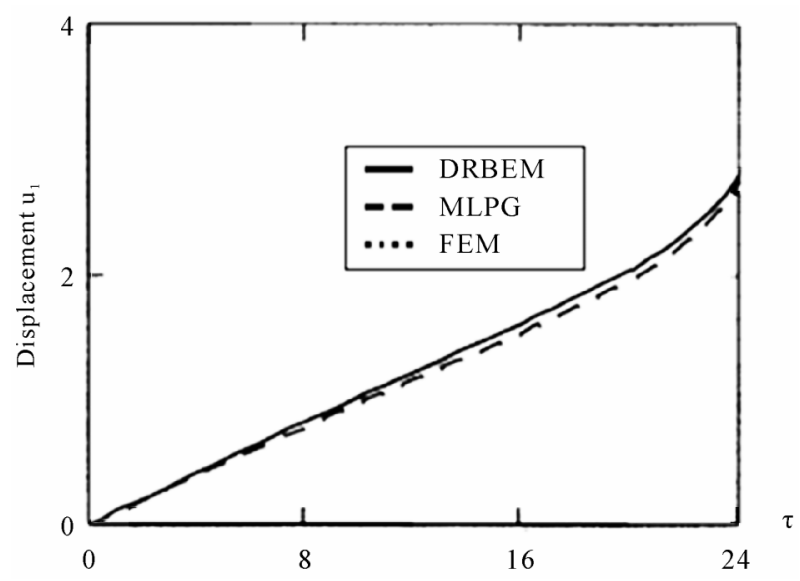

Figure 8. Variation of the displacement $u_{1}$ with time $\tau$ for the three methods: DRBEM, MLPG and FEM.

$\sigma_{12}$ and $\sigma_{22}$ are closer in the absence of initial stress than in the presence of initial stress. We may also observe from these figures that the initial stress has an important effect on the magneto-thermo-visco-elastic stresses along $x$-axis through the material constants, thermal constants, magnetic constants and viscosity factor, which are essential parameters to be considered in the design of various devices. Furthermore, while there is no limitation in the solution procedure, all boundary and initial conditions are strongly satisfied.

This phenomenon gives clear evidence of a magnetothermostress-focusing effect in a non-homogeneous anisotropic initially stressed viscoelastic solid. From this knowledge of the variation of magneto-thermo-viscoelastic stresses along $x$-axis in a non-homogeneous anisotropic initially stressed viscoelastic solid placed in a constant primary magnetic field, we can design various viscoelastic solids under magnetothermal load to meet specific engineering requirements and utilize it in meas- urement techniques of thermoviscoelasticity.

\section{Conclusions}

The purpose of this paper is to investigate the transient magneto-thermo-visco-elastic stresses in a non-homogeneous anisotropic body. A development of the dual reciprocity boundary element method for solving the system of fundamental equations is presented. In the case of plane deformation, a numerical scheme for the implementation of the method is presented and the numerical computations are carried out for the temperature, displacement components and thermal stress components. The validity of DRBEM is examined by considering a magneto-thermo-visco-elastic solid occupies a rectangular region and excellent agreement is obtained with existent results. The results obtained are presented graphically to show the effects of inhomogeneity and initial stress on the displacement components and thermal stress components.

\section{References}

[1] A. M. El-Naggar, A. M. Abd-Alla and M. A. Fahmy, "The Propagation of Thermal Stresses in an Infinite Elastic Slab," Applied Mathematics and Computation, Vol. 157, No. 2, 2004, pp. 307-312. doi:10.1016/j.amc.2003.08.116

[2] A. M. El-Naggar, A. M. Abd-Alla, M. A. Fahmy and S. M. Ahmed, "thermal Stresses in a Rotating Non-Homogeneous Orthotropic Hollow Cylinder," Heat and Mass Transfer, Vol. 39, No. 1, 2002, pp. 41-46. doi:10.1007/s00231-001-0285-4

[3] C. A. Brebbia and D. Nardini, "Dynamic Analysis in Solid Mechanics by an Alternative Boundary Element Procedure," International Journal of Soil Dynamics and Earthquake Engineering, Vol. 2, No. 4, 1983, pp. 228-233. doi:10.1016/0261-7277(83)90040-2

[4] L. C. Wrobel and C. A. Brebbia, "The Dual Reciprocity Boundary Element Formulation for Nonlinear Diffusion Problems," Computer Methods in Applied Mechanics and Engineering, Vol. 65, No. 2, 1987, pp. 147-164. doi:10.1016/0045-7825(87)90010-7

[5] P. W. Partridge, C. A. Brebbia and L. C. Wrobel, "The Dual Reciprocity Boundary Element Method," Computational Mechanics Publications, Southampton, 1992.

[6] E. A. Divo and A. J. Kassab, "Boundary Element Methods for Heat Conduction: With Applications in Non-Homogeneous Media," WIT Press, Southampton, 2003.

[7] L. Gaul, M. Kögl and M. Wagner, "Boundary Element Methods for Engineers and Scientists," Springer-Verlag, Berlin, 2003.

[8] T. Matsumoto, A. Guzik and M. Tanaka, "A Boundary Element Method for Analysis of Thermoelastic Deforma- 
tions in Materials with Temperature Dependent Properties," International Journal for Numerical Methods in Engineering, Vol. 64, No. 11, 2005, pp. 1432-1458. doi:10.1002/nme.1412

[9] M. A. Fahmy, "Thermoelastic Stresses in a Rotating Nonhomogeneous Anisotropic Body," Numerical Heat Transfer, Part A: Applications, Vol. 53, No. 9, 2008, pp. 10011011. doi:10.1080/10407780701789179

[10] M. A. Fahmy, "Application of DRBEM to Non-Steady State Heat Conduction in Non-Homogeneous Anisotropic Media under Various Boundary Elements," Far East Journal of Mathematical Sciences, Vol. 43, 2010, pp. 83-93.

[11] M. A. Fahmy, "A Time-Stepping DRBEM for the Transient Magneto-Thermo-Visco-Elastic Stresses in a Rotating Non-Homogeneous Anisotropic Solid," Engineering Analysis with Boundary Elements, Vol. 36, 2012, pp. 335- 345. doi:10.1016/j.enganabound.2011.09.004

[12] G. Davì and A. Milazzo, "A Regular Variational Boundary Model for Free Vibrations of Magneto-Electro-Elastic Structures," Engineering Analysis with Boundary Elements, Vol. 35, No. 3, 2011, pp. 303-312. doi:10.1016/j.enganabound.2010.10.004

[13] M. A. Fahmy and T. M. El-Shahat, "The Effect of Initial Stress and Inhomogeneity on the Thermoelastic Stresses in a Rotating Anisotropic Solid," Archive of Applied Mechanics, Vol. 78, No. 6, 2008, pp. 431-442. doi:10.1007/s00419-007-0150-0

[14] C. A. Brebbia, J. C. F. Telles and L. Wrobel, "Boundary Element Techniques in Engineering," Springer-Verlag, New York, 1984.

[15] P. W. Partridge and L. C. Wrobel, "The Dual Reciprocity Boundary Element Method for Spontaneous Ignition," International Journal for Numerical Methods in Engineering, Vol. 30, No. 5, 1990, pp. 953-963. doi:10.1002/nme. 1620300502

[16] D. Nardini and C. A. Brebbia, "A New Approach to Free Vibration Analysis Using Boundary Elements," Boundary Element Methods, In: C. A. Brebbia, Ed., SpringerVerlag, Berlin, 1982, pp. 312-326.

[17] E. L. Albuquerque, P. Sollero and M. H. Aliabadi, "Dual Boundary Element Method for Anisotropic Dynamic Fracture Mechanics," International Journal for Numerical Methods in Engineering, Vol. 59, No. 9, 2004, pp. 1187-1205. doi:10.1002/nme.912

[18] H. A. Cho, M. A. Golberg, A. S. Muleshkov and X. Li, "Trefftz Methods for Time Dependent Partial Differential Equations," CMC-Computers, Materials \& Continua, Vol. 1, 2004, pp. 1-37.
[19] M. Guiggiani and A. Gigante, "A General Algorithm for Multidimensional Cauchy Principal Value Integrals in the Boundary Element Method," Journal of Applied Mechanics, ASME, Vol. 57, 1990, pp. 906-915. doi:10.1115/1.2897660

[20] V. Mantič, "A New Formula for the C-Matrix in the Somigliana Identity," Journal of Elasticity, Vol. 33, 1993, pp. 191-201. doi:10.1007/BF00043247

[21] G. H. Golub and C. F. Van Loan, "Matrix Computations," North Oxford Academic, Oxford, 1983.

[22] K. J. Bathe, "Finite Element Procedures," Prentice-Hall, Englewood Cliffs, 1996.

[23] N. Ahmed, "Visco-Elastic Boundary Layer Flow Past a Stretching Plate and Heat Transfer with Variable Thermal Conductivity," World Journal of Mechanics, Vol. 1, No. 3, 2011, pp. 15-20. doi:10.4236/wjm.2011.12003

[24] S. Kanaun, "An Efficient Numerical Method for Calculation of Elastic and Thermo-Elastic Fields in a Homogeneous Medium with Several Heterogeneous Inclusions," World Journal of Mechanics, Vol. 1, No. 2, 2011, pp. 31-43. doi:10.4236/wjm.2011.12005

[25] M. D. Monsia, “A Simplified Nonlinear Generalized Maxwell Model for Predicting the Time Dependent Behavior of Viscoelastic Materials," World Journal of Mechanics, Vol. 1, No. 3, 2011, pp. 158-167. doi:10.4236/wim.2011.13021

[26] A. M. Abd-Alla, T. M. El-Shahat and M. A. Fahmy, "Thermoelastic Stresses in Inhomogeneous Anisotropic Solid in the Presence of Body Force," International Journal of Heat \& Technology, Vol. 25, No. 1, 2007, pp. 111-118.

[27] A. M. Abd-Alla, M. A. Fahmy and T. M. El-Shahat, "Magneto-Thermo-Elastic Stresses in Inhomogeneous Anisotropic Solid in the Presence of Body Force," FarEast Journal of Applied Mathematics, Vol. 27, No. 3, 2007, pp. 499-516.

[28] A. M. Abd-Alla, M. A. Fahmy and T. M. El-Shahat, "Magneto-Thermo-Elastic Problem of a Rotating Non-Ho- mogeneous Anisotropic Solid Cylinder," Archive of Applied Mechanics, Vol. 78, No. 2, 2008, pp. 135-148. doi:10.1007/s00419-007-0147-8

[29] J. Sladek, V. Sladek, P. Solek and Ch. Zhang, "Fracture Analysis in Continuously Nonhomogeneous MagnetoElectro-Elastic Solids under a Thermal Load by the MLPG," International Journal of Solids and Structures, Vol. 47, No. 10, 2010, pp. 1381-1391. doi:10.1016/j.ijsolstr.2010.01.025 